\title{
Siphonic Removal of Sediments in Water Reservoirs - Additional Experiment for Model Revision
}

\author{
M. Sadatomi, T. Nagano, and A. Kawahara
}

\begin{abstract}
In water reservoirs and dams in Japan, the increment of sediments causes capacity reduction and water quality deterioration. In the present paper, the siphonic sediment removal system, invented by Sadatomi, is experimentally studied using different sized siphons and sands. From the present and our previous data for spherical particles, a new correlation of particle volume flow rate fraction is obtained. By incorporating the new correlation, the performance prediction model proposed in our previous study is revised. The revised model is validated by testing against the present data as well as previous data. Thus, the revised model is promising as a design tool of actual large scale systems.
\end{abstract}

Index Terms-Removal, sediments, siphon, water reservoirs.

\section{INTRODUCTION}

In water reservoirs and dams in Japan, the increment of sediments on their beds causes the reduction of capacity and the deterioration of water quality. However, commonly used removal system, such as sand pump [1] and bucket [2], requires a large power and cost. Thus, the development of a new efficient and economic system for removing sediments is eagerly desired. Such a situation provoked Sadatomi to invent a new siphonic removal system with a little power except for the first operation [3]. Two representative siphonic removal systems in Japan are: (a) the sediments and water are sucked by a siphon pipe connected to a closed water tank by pumping water from the tank to an another pipe [4]; (b) the sediments and water are sucked by a single siphon pipe penetrating horizontally the dam, and the inlet of the siphon are hanged up and down to prevent the occlusion of the sediments [5]. The first type has the following two demerits: (a) a large power is required for the pumping; (b) a closed tank and a large separator tank must be submerged in the reservoir thus high cost. The second type has the following two demerits: (a) it is not easy to penetrate the siphon pipe for a used dam; (b) it is ineffective because the time of sucking water alone is much longer than that of both the sediments and water. Contrary to these, Sadatomi's new siphon system [3] can continuously remove sediments with a small amount of water because the siphon inlet automatically drop with the remove of sediments, and has merits that it need a little power except for the first operation and that the maintenance and the

Manuscript received March 20, 2014; revised July 11, 2014.

M. Sadatomi and A. Kawahara are with the Dept. of Advanced Mechanical Systems, Graduate School of Science and Technology, Kumamoto University, Kumamoto, Japan (e-mail: sadatomi@mech.kumamoto-u.ac.jp, akimaro@mech.kumamoto-u.ac.jp).

T. Nagano is with Mitsubishi Electric Co., Wakayama, Japan (e-mail: dir25122@yahoo.co.jp). control of the siphon are easy for its simple structure.

In our previous study [6], experiments were conducted using spherical particles as the sediments and 20 and $30 \mathrm{~mm}$ i.d. small scale siphons with square-edged inlet as the test siphon. In addition, a simple one-dimensional model was proposed to predict the performance of the siphon which is needed to design a large scale siphon in actual reservoirs. The model was validated against the data. However, experiments using non-spherical particles, which are important in actual use, have never been conducted.

In the present study, therefore, experiments have been conducted using two-different size sands and spherical particles as the test particles and 30 and $40 \mathrm{~mm}$ i.d. middle scale siphons with square-edged and rounded inlets as the test siphon. Furthermore, the performance prediction model [6] has been revised and tested against the data in the present experiments and the data in the previous paper. The results of such experiments and model tests are described in the present paper.

\section{SiPHON System by SADATOMI [3]}

Fig. 1 shows the siphon system invented by Sadatomi. The suction part consists of inner siphon pipe and sliding outer pipe with a flange. The sliding outer pipe can automatically drop by its weight and a negative pressure arisen between the flange and the sediments when the sediments are sucked by the siphon. Thus, the distance from the flange to the sediments is properly kept, and the sediments can be continuously and efficiently removed. In addition to the drop of the sliding outer pipe, the inlet of the siphon pipe can be placed in wide area by horizontal rails on the bank and horizontal arm for rotation, and so on. In the first operation of the system, water are poured from the top by opening the valve 3 at the close of valves 1 and 2, then the valve 3 is closed and valves 2 and 1 are opened in turn.

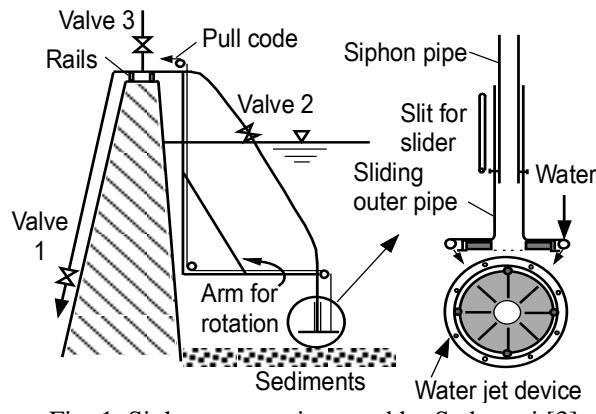

Fig. 1. Siphon system invented by Sadatomi [3].

The bottom part of sliding outer tube consists of a circular flange, a rough screen having the same diameter as the flange, 
baffle plates and water jet device if necessary. The screen prevents the occlusion of the siphon by the entrance of large stone etc. The baffle plate prevents the generation of free vortex causing centrifugal force in the siphon pipe. Thus the sands and mud can flow in the central core of the siphon, minimizing the friction between them and the pipe wall. The water jet device is necessary when the sediments are hard adhesive clay because the clay must be broken in order to introduce water into the siphon pipe.

\section{EXPERIMENT}

Fig. 2 shows the test apparatus, and two types of siphon inlet: the square-edged inlet [6] and the rounded inlet which was newly tested. The curvature radius of the rounded inlet was $7.5 \mathrm{~mm}$ and $10.0 \mathrm{~mm}$ respectively for $30 \mathrm{~mm}$ and $40 \mathrm{~mm}$ i.d. siphon pipes, being proportional to the siphon radius.

A $0.5 \mathrm{~m}$ i.d. and $1.5 \mathrm{~m}$ deep tank, i.e. an upper tank, was filled with test particles and tap water, and was placed on the second floor of our laboratory. From the tank, the particles and water flowed up, flowed horizontally above a handrail, and flowed down into a separator. In the separator, the particles were separated from water with a net and discharged into a bucket on an electric balance. The volume flow rate of particles, $Q_{S}$, was determined from the time variation of particles weight in the bucket and the particle density. The volume flow rate of water after separation, $Q_{L}$, was measured with an electromagnetic flow meter. The particles volume fraction in the siphon pipe, $\alpha_{S}$, was measured with a constant electric current method developed by Fukano [7]. In order to keep the water level in the upper tank constant, water with the same volume as the total of water and particles discharged was returned to the upper tank with a centrifugal pump.
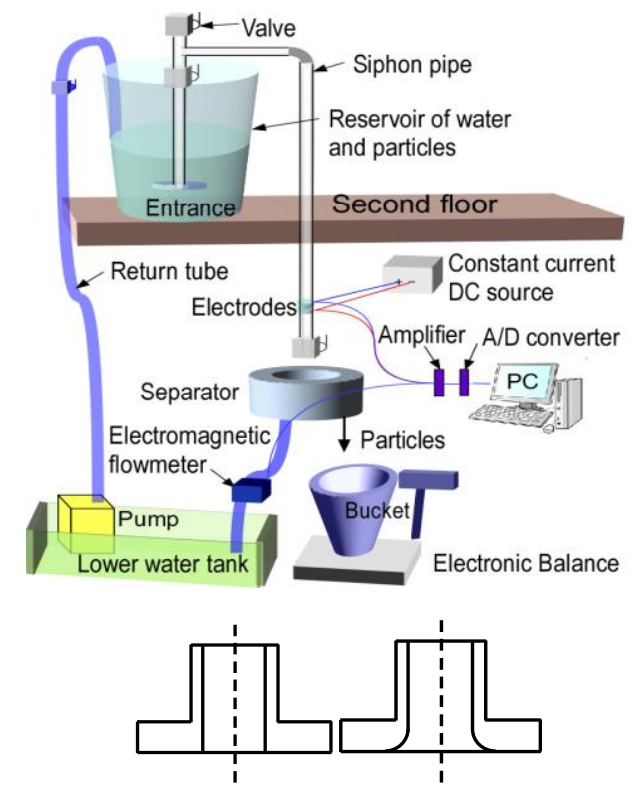

Fig. 2. Test apparatus, and square-edged and rounded inlets of sliding outer tube.

Fig. 3 (a) shows picture of a full view of the present test apparatus. A union joint and a lower pipe below the joint seen in the picture center allow us to change the vertical level difference from the water surface of the upper tank to the siphon exit, $H$, i.e. the driving head of the siphon system. Fig.
3 (b) shows picture of a typical SP-2 particles-water down flow in $30 \mathrm{~mm}$ i.d. siphon pipe with the square-edged inlet. As you can see, the particles homogeneously distributed in both radial and axial direction of the siphon pipe, and flowed down steadily.

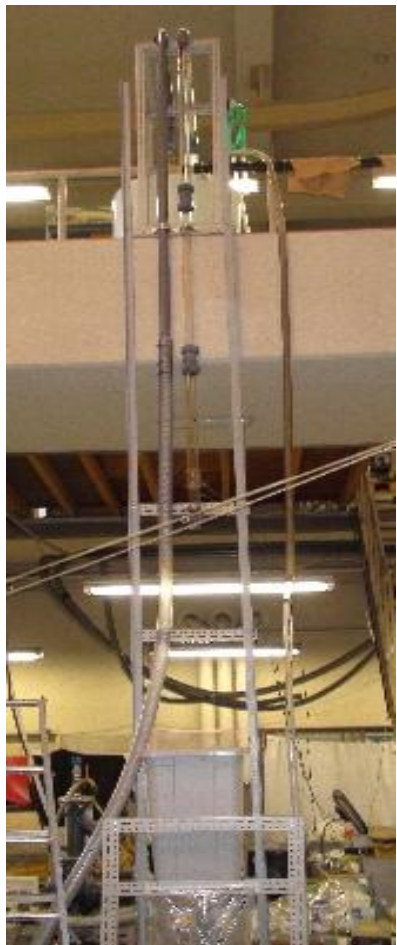

(a)

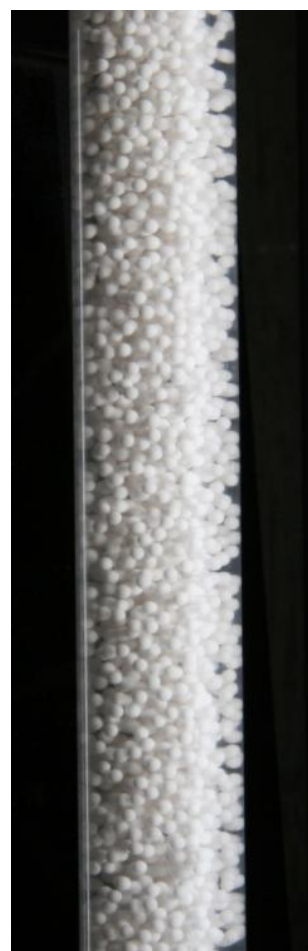

(b)
Fig. 3. Pictures of test apparatus and typical down flow in $30 \mathrm{~mm}$ i.d. siphon (SP-2, Square-edged inlet).

Since the particles in the upper tank were not enough even at the beginning of the experiment, the duration of the steady flow in the siphon was not so long. So, in order to obtain the steady flow data of $Q_{S}, Q_{L}$ and $\alpha_{S}$, the output signals from the respective detectors were fed to a personal computer via a data acquisition system, and the respective time mean values were adopted as the experimental data. The duration of the steady flow became shorter with increasing of the driving head, $H$, because $Q_{S}$ and $Q_{L}$ increased with the head. The uncertainties of measurements were estimated to be within about $5 \%$ for $Q_{S}$ and $Q_{L}$, and $3 \%$ for $\alpha_{S}$.

Table I lists the specifications of four kinds of the siphon pipe: two small type siphons used in our previous study [6] and two medium ones in the present study. The total length from the inlet to the exit of the siphon, $L$, the horizontal length connecting the upriser pipe and the downcomer pipe, $L_{H}$, and the level difference, $H$, are listed.

TABLE I: SPECIFICATION OF FOUR KINDS OF SIPHON

\begin{tabular}{|c|c|c|c|c|}
\hline $\begin{array}{c}\text { Name of } \\
\text { system }\end{array}$ & $\begin{array}{c}\text { Diameter } \\
d(\mathrm{~mm})\end{array}$ & $\begin{array}{l}\text { Total } \\
\text { length } \\
L(\mathrm{~m})\end{array}$ & $\begin{array}{c}\text { Horizontal } \\
\text { length } \\
L_{H}(\mathrm{~m})\end{array}$ & $\begin{array}{c}\text { Level } \\
\text { difference } \\
H(\mathrm{~m})\end{array}$ \\
\hline Small & $20 \& 30$ & 2.8 to 3.3 & 0.4 & 1.5 to 2.0 \\
\hline Medium & $30 \& 40$ & 5.1 to 6.1 & 1.1 & 2.5 to 3.5 \\
\hline
\end{tabular}

Table II lists the specifications of test particles. Spherical particles of SP-1, SP-2, SP-4, and SP-1 \& SP-4 mixture were the same as those used in our previous study for the small type siphons [6]. In addition to these, non-spherical ones, Sand-S and Sand-L (larger particles selected by screening 
from K3 and K4 type in Kumamoto-Silica Mining Co. Ltd.), were used in the present experiments. Mean diameter, material, and mean density of each particle are listed. As to the density, SP-1 and SP-2 were about 1.4 times of the sand.

TABLE II: SPECIFICATIONS OF PARTICLES

\begin{tabular}{|c|c|c|c|}
\hline Name & $\begin{array}{c}\text { Mean dia. } \\
(\mathrm{mm})\end{array}$ & Materials & $\begin{array}{c}\text { Mean density } \\
\left(\mathrm{kg} / \mathrm{m}^{3}\right)\end{array}$ \\
\hline SP-1 & 1.2 & Aluminum oxide & 3760 \\
\hline SP-2 & 2.1 & Aluminum oxide & 3690 \\
\hline SP-4 & 4.0 & Ceramics & 2600 \\
\hline $\begin{array}{c}\text { SP-1 \& SP-4 } \\
\text { mixture* }\end{array}$ & 1.3 & $\begin{array}{c}\text { Aluminum oxide } \\
\text { \& Ceramics }\end{array}$ & 3160 \\
\hline Sand-S & 1.6 & Sand & 2600 \\
\hline Sand-L & 3.4 & Sand & 2600 \\
\hline
\end{tabular}

* 51.3 vol. $\%$ for SP-1

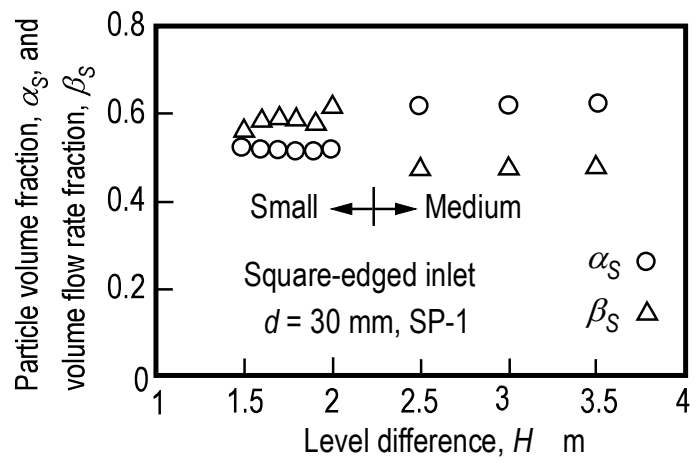

(a) SP-1

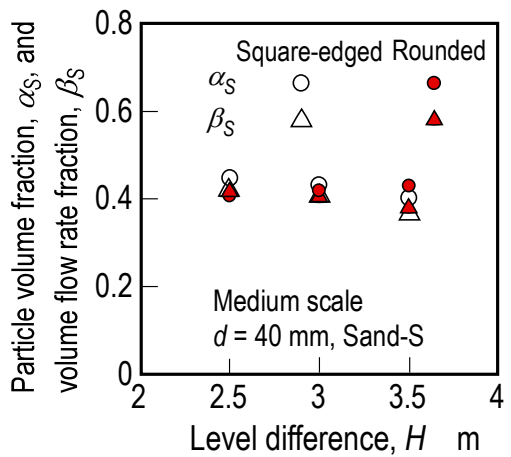

(b) Sand-S

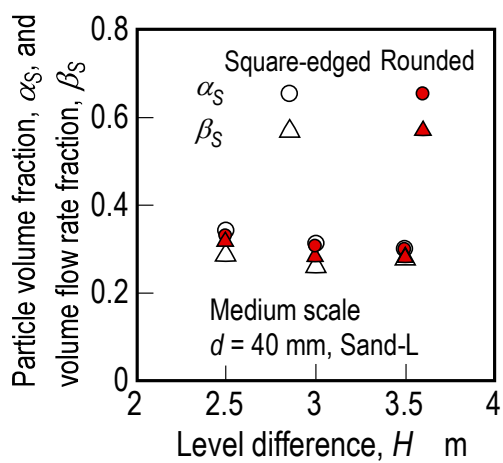

(c) Sand-L

Fig. 4. Comparison between particle volume fraction and particle volume flow rate fraction.

\section{EXPERIMENTAL RESULTS}

Sadatomi et al. [6] proposed a performance prediction model of the present siphon system, by assuming the particles-water flow in the siphon as a homogeneous mixture flow. In order to confirm the validity of the assumption, the particle volume fraction data, $\alpha_{S}$, are compared with the particle volume flow rate fraction data, $\beta_{S}$, which was determined by substituting the measured volume flow rates of water and particles into $\beta_{S}=Q_{S} /\left(Q_{L}+Q_{S}\right)$. Fig. 4(a) - (c) shows such comparison results for the spherical SP-1, non-spherical Sand-S, and Sand-L. $\alpha_{S}$ data are plotted as circular symbols, while $\beta_{S}$ data as triangular symbols. The abscissa is the level difference, $H$. In Fig. 4(a) for SP-1 particles in $30 \mathrm{~mm}$ i.d. siphon with square-edged inlet, $\beta_{S}>$ $\alpha_{S}$ in the small type siphon while $\alpha_{S}>\beta_{S}$ in the medium type one. The difference in data trend is not only caused by the difference of $H$ but also by that of the horizontal siphon length, $L_{H}$. $L_{H}$ in the medium type siphon is three times longer than that in the small type one, thus particles in the medium type one mainly flow in the bottom part of the pipe cross-section, thus the particles velocity becomes slow, and $\alpha_{S}$ becomes high even in the downcomer. In Fig. 4(b) and (c) for Sand-S and Sand L in $40 \mathrm{~mm}$ i.d. siphon, however, the difference between $\alpha_{S}$ and $\beta_{S}$ is quite small, irrespective of inlet geometry. Thus, the assumption of homogeneous mixture flow in the siphon, i.e., $\alpha_{S}=\beta_{S}$, is acceptable for non-spherical sands which are more important in practical applications.

In order to efficiently remove the sediments, the ratio of the sediments to water in the siphon is higher the better. Since the present siphon system has a beneficial function that the siphon inlet can slide down with the removal of the sediments, $\alpha_{S}$ value is quite high and between 0.3 and 0.6 as seen in Fig. 4(a) - (c). $\alpha_{S}$ value is higher for the spherical SP-1 than that for the non-spherical Sand-S and Sand-L, and $\alpha_{S}$ value for Sand-S is higher than that of Sand-L. The presumable reason of these is that $\alpha_{S}$ value when the particles are well packed in the same siphon pipe by hand is higher in SP-1 than Sand-S, and is higher in Sand-S than Sand-L.

\section{EXAmination OF PERFormance Prediction Model}

Similarly to our previous paper [6], the performance of the siphon has been predicted by assuming the particles and water flow in the siphon as the homogeneous mixture flow. In the present model, however, the correlation of $\beta_{S}$ has been revised in order to widen the applicability to the non-spherical sands and to the rounded siphon.

The basic equation of the model is the conservation equation of energy between the water surface of the upper tank and the exit of the siphon:

$$
\rho_{L} g H=\rho_{H} \frac{u_{H}^{2}}{2}+\left(\zeta_{I}+2 \zeta_{E}+\lambda \frac{L}{d}\right) \rho_{H} \frac{u_{H}^{2}}{2}+\zeta_{P} \rho_{L} \frac{u_{L e}^{2}}{2}
$$

Here, the left-hand-side of Eq. (1) is the hydrostatic pressure when the siphon exit is taken as the base level. The three terms of the right-hand-side are respectively the dynamic pressure of the homogeneous mixture flow, the total of the pressure loss at siphon inlet, two elbows, and wall friction, and the pressure loss in the sediment bed. In Eq. (1), $\rho$ is the density, $u$ the mean velocity, $H$ the level difference between the water surface of the upper tank and the siphon exit, $L$ and $d$ the total length and the diameter of the siphon 
pipe. In the present calculation, the friction factor, $\lambda$, was calculated from the Blasius' equation:

$$
\lambda=0.3164\left(\mu_{H} / \rho_{H} u_{H} d\right)^{0.25} .
$$

Here, the density of the homogeneous mixture was taken as $\rho_{H}=\left(\rho_{L} Q_{L}+\rho_{S} Q_{S}\right) /\left(Q_{L}+Q_{S}\right)$, and the viscosity of that as $\mu_{H}=\mu_{L}$ [8]. The siphon inlet loss coefficient, $\zeta_{I}$, and the pressure loss coefficient in the sediment bed, $\zeta_{P}$, were determined by preliminary experiments as described in Appendix. The elbow loss coefficient, $\zeta_{E}$, was taken as $\zeta_{E}=$ $0.21[9]$.

If the water velocity through sediment bed is assumed to be equal to the homogeneous mixture velocity, i.e., $u_{L e}=u_{H}$, the mixture velocity can be calculated by

$$
u_{H}=\sqrt{\frac{2 \rho_{L} g H}{\rho_{H}\left(1+\zeta_{I}+2 \zeta_{E}+\lambda \frac{L}{d}\right)+\zeta_{P} \rho_{L}}}
$$

In the calculation of Eq. (3), an iteration technique is needed because $\lambda$ is a function of $u_{H}$ as seen in Eq. (2).

Since the solid-liquid mixture is assumed to be homogeneous, the liquid and solid volumetric fluxes are given by

$$
j_{L}=u_{H}\left(1-\beta_{S}\right), j_{S}=u_{H} \beta_{S}
$$

By multiplying them to the siphon cross-sectional area, $A$, the volume flow rates of liquid and solid are given by

$$
Q_{L}=u_{H}\left(1-\beta_{S}\right) A, Q_{S}=u_{H} \beta_{S} A .
$$

Thus, if $\beta_{S}$ can be correlated as a function of flow parameters, $Q_{L}$ and $Q_{S}$ are calculated from Eqs. (2) - (5).

In our previous study [6] for spherical particles-water flows in the siphon with the square-edged inlet, we proposed a $\beta_{S}$ correlation as follows:

$$
\beta_{S}=0.723 e^{-5.27 \frac{d_{P}}{d}} s^{0.03}\left(\frac{u_{P} d_{P}}{v_{W}}\right)^{-0.02}
$$

Here, $d_{P}$ is the mean particle diameter, $s$ the specific gravity of the particle, $u_{P}$ the settling velocity of the single particle in a stagnant water pool (measured value), and $v_{W}$ the kinematic viscosity of water.

In the present study, by accounting for the effects of the horizontal siphon length, $L_{H}$, a new $\beta_{S}$ correlation is proposed:

$$
\beta_{\mathrm{S}}=K\left(\frac{d_{P}}{d}\right)^{-0.2}\left(\frac{L_{H}}{d}\right)^{-0.07}\left(\frac{u_{P} d_{P}}{v_{W}}\right)^{-0.2}
$$

Here, the experimental constant, $K$, is 1.09 and 0.86 respectively for spherical and non-spherical particles in the square-edged siphon inlet, while 1.17 and 0.85 for those in the rounded siphon nlet.

Fig. 5 (a) and Fig. 5 (b) compare $\beta_{S}$ values between experiment and calculation respectively by Eqs. (6) and (7). The data are the mean $\beta_{S}$ value at different level differences, $H$, in each particle. The data are plotted as darkened symbols for the small type siphon and open symbols for the middle type one with different symbols for each particle. In addition, the data for the rounded inlet are marked with + on the respective symbols. The calculation by Eq. (6) over-predicts the data about $15 \%$ as seen in Fig. 5 (a), while that by Eq. (7) fits well the data as seen in Fig. 5 (b). Thus, an improvement of the predictability by Eq. (7) is confirmed.

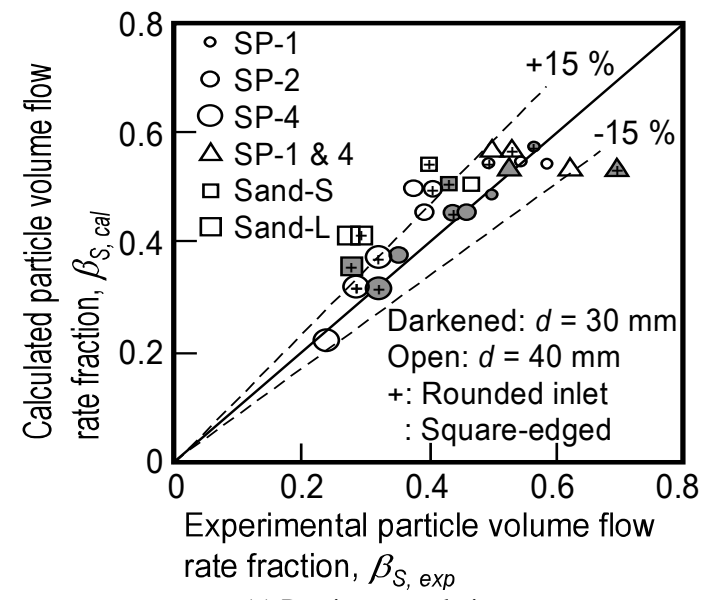

(a) Previous correlation

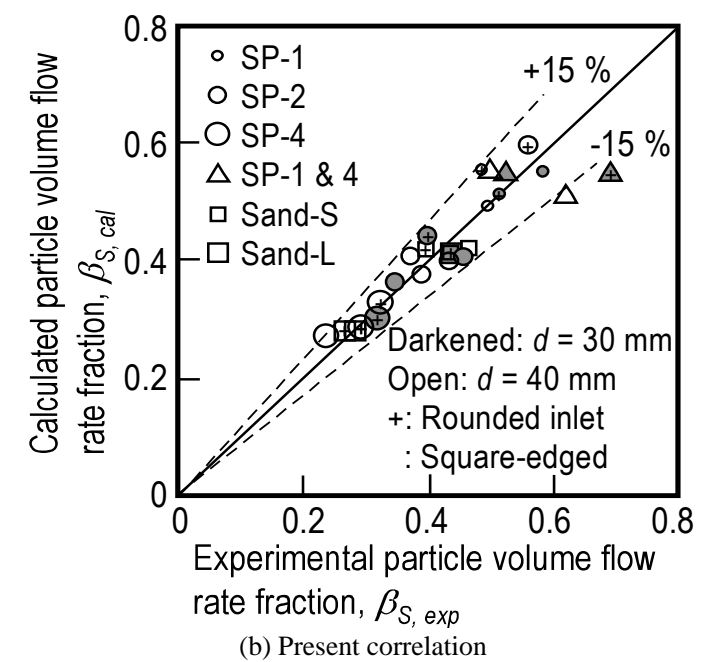

Fig. 5. Comparison of particle volume flow rate fraction between experiment and calculation.

The discharge rates of water and particles, $Q_{L}$ and $Q_{S}$, were calculated by the model [6] with Eq. (6) and/or Eq. (7). The calculated results are compared with the data for Sand-S in the medium type siphon in Fig. 6 (a), Fig. 6(b) and for Sand-L in the small type siphon in Fig. 6 (c), Fig. 6(d), respectively for the square-edged and the rounded inlets. $Q_{L}$ and $Q_{S}$ data are plotted with circular and triangular symbols, while the calculations with Eq. (6) and Eq. (7) are drawn as hair lines and heavy lines. These comparisons justified that the calculations with Eq. (7) are superior to those with Eq. (6) regardless of the siphon type, the size of the sands, and the geometry of the siphon inlet.

The calculated results by the model [6] with Eq. (7) are also tested against the data for spherical particles of SP-1 and SP-4 in the medium type siphon. Fig. 7 (a) and Fig. 7 (b) show the results respectively for $40 \mathrm{~mm}$ and $30 \mathrm{~mm}$ i.d. 
siphons with the rounded inlet. The data for SP-4 and SP-1 are plotted as circular and triangular symbols, and $Q_{S}$ and $Q_{L}$ data are shown as red and open symbols. The calculated results of $Q_{S}$ and $Q_{L}$ are drawn as red solid curves and black broken curves with hair line and heavy line respectively for SP-4 and SP-1. We can conclude that the agreement between the data and the calculation is better for $Q_{S}$ than $Q_{L}$, and the agreement for $Q_{S}$ is better in SP-1 than SP-4, and that for $Q_{L}$ is better in SP-4 than SP-1.

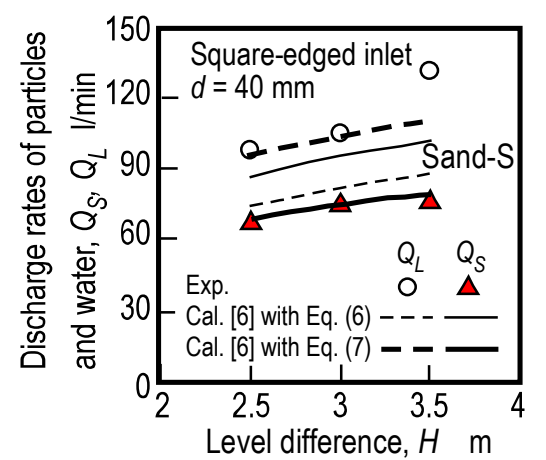

(a) $d=40 \mathrm{~mm}$, Square-edged inlet, Sand-S

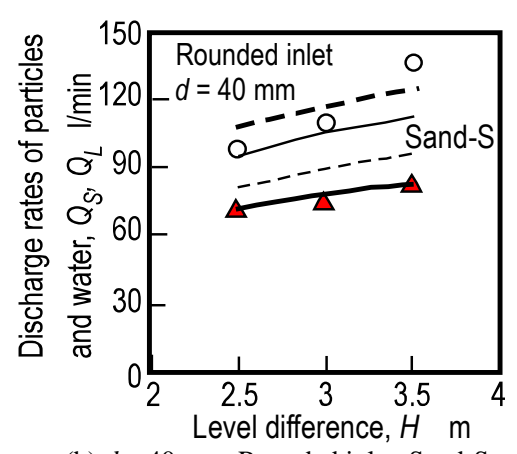

(b) $d=40 \mathrm{~mm}$, Rounded inlet, Sand-S

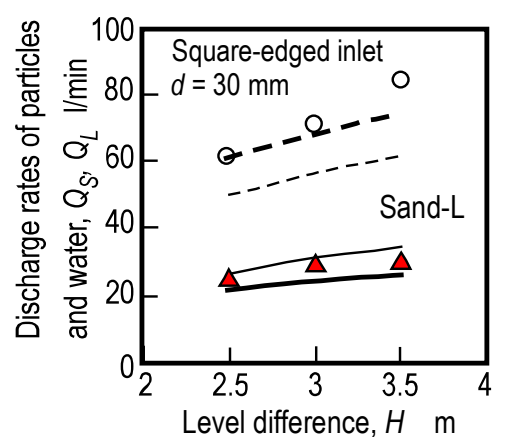

(c) $d=30 \mathrm{~mm}$, Square-edged inlet, Sand-L

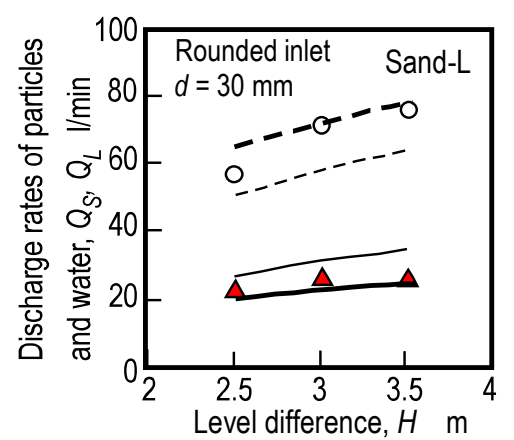

(d) $d=30 \mathrm{~mm}$, Rounded inlet, Sand-L

Fig. 6. Comparison of liquid and solid flow rates between experiment and calculation when Eq. (6) and Eq. (7) are used for Sand-S and Sand L.

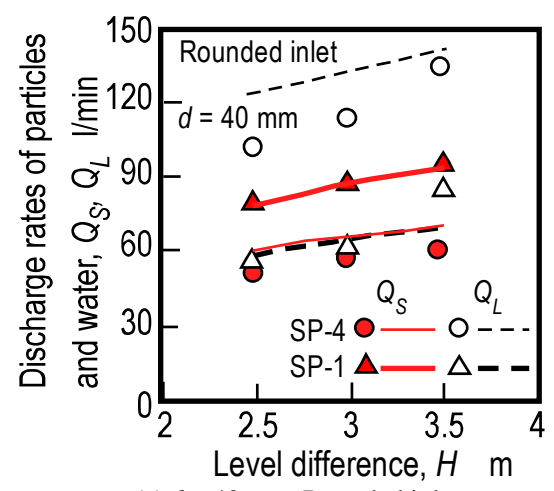

(a) $d=40 \mathrm{~mm}$, Rounded inlet

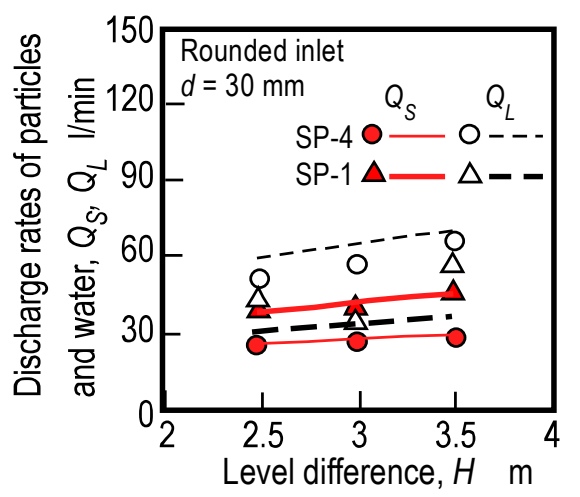

(b) $d=30 \mathrm{~mm}$, Rounded inlet

Fig. 7. Comparison of liquid and solid flow rates between experiment and calculation when Eq. (7) is used for SP-1 and SP-4.

\section{SUMMARY AND CONCLUSIONS}

In order to develop an efficient and economic siphon system to remove sediments on reservoir beds, experiments were conducted using 30 and $40 \mathrm{~mm}$ i.d siphons in laboratory scale and two-different size sands and spherical particles. In addition, the performance prediction model proposed in our previous study [6] was revised by considering the effects of the size and the geometry of both the siphon and the particles. The followings are main findings:

1) The particle volume fraction in the siphon, $\alpha_{S}$, was from 0.3 to 0.6 , being the evidence of very high sediments suction performance.

2) Since $\alpha_{S}$ was nearly equal to $\beta_{S}$, the water-particles mixture in the siphon can be regarded as homogeneous. However, the relationship between them depended on the horizontal siphon length. So, a new $\beta_{S}$ correlation accounting for the length was developed.

3) By incorporating the new $\beta_{S}$ correlation into the model [6], the model can predict well the present particles and water discharge data regardless of both the siphon and particles size and geometry.

\section{APPENDIX: EVALUATION OF $\zeta_{I}$ AND $\zeta_{P}$}

The siphon inlet pressure loss coefficient, $\zeta_{I}$, was determined by measuring the water volume flow rate, $Q_{L}$, when no particles was stocked in the upper tank, and fitting the measured $Q_{L}$ to the calculated one from Eqs. (3) - (5) by taking $\rho_{H}=\rho_{L}$ and $\beta_{S}=0$. In the fitting, the calculation was iterated by changing $\zeta_{I}$ value, and $Q_{L}$ value was compared between the measurement and the calculation. $\zeta_{I}$ value shows 
best agreement between them was selected as the loss coefficient. The best fit value of $\zeta_{I}$ written on Fig. 8 is higher for the $30 \mathrm{~mm}$ i.d. siphon than the $40 \mathrm{~mm}$ one, and that it is higher for the square-edged inlet than the rounded one. Furthermore, the present $\zeta_{I}$ values depend on the siphon pipe diameter and are larger than those listed in common text book of "Fluid Mechanics." The reason is probably that the flange diameter and the roughness are different between them.

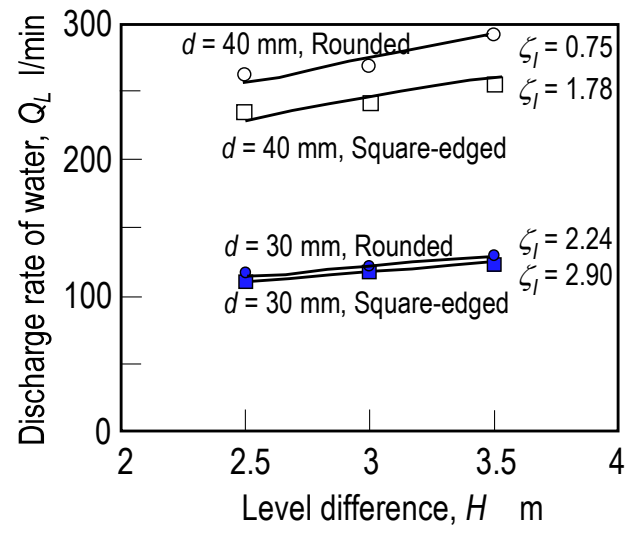

Fig. 8. Comparison of liquid flow rate between experiment and calculation by Eq. (1) when $\zeta_{I}$ written on figure was used for Single-phase water flows.

The pressure loss coefficient in the particles bed, $\zeta_{P}$, was determined by measuring the water volume flow rate, $Q_{L}$, by covering the siphon inlet with a thin stocking reinforced with a rough screen, in order to block the suction of particles [6]. Since the resulting $\zeta_{P}$ value was almost independent of the kinds of the present particles, the averaged value of $\zeta_{P}=2.6$ was adopted in the performance prediction, irrespective of the particles.

\section{NOMENCLATURE}

$A:$ cross-section area of siphon pipe
$d:$ inner diameter of siphon pipe
$L:$ length of siphon pipe
$g:$ gravitational acceleration
$H:$ level difference
$j:$ volumetric flux
$K$ : constant in Eq. $(7)$
$Q:$ volume flow rate
$u$ : velocity

\section{Greek letters}

$\alpha$ : volume fraction

$\beta$ : volume flow rate ratio

$\rho$ : density

$\lambda$ : friction coefficient for pipe wall

$\zeta_{i}$ inlet loss coefficient

$\zeta_{P}$ : loss coefficient for water flow through particles bed

Subscripts

$L$ : liquid phase
$L e$ : effective liquid

$S$ : solid phase

$H$ : homogeneous mixture or horizontal

\section{ACKNOWLEDGMENT}

The authors wish to appreciate Mr. G. Ariyoshi, technician at the Faculty of Engineering, Kumamoto University, for his cooperation of manufacturing experimental apparatus, and $\mathrm{T}$. Kubota and T. Kusano, undergraduate students in those days at Kumamoto University, for their experimental cooperation.

\section{REFERENCES}

[1] S. Hamada, "Dredging method and dredging system," Japanese Patent JP, 2002-266369, A, September 18, 2002.

[2] K. Mishima, "Stationary and movable bucket dredging system," Japanese Patent JP, 2001-262613, A, September 26, 2001.

[3] M. Sadatomi, "Sediments removal system," Japanese Patent JP 2008-223465, A, September 25, 2008.

[4] S. Oigawa, "Dredging method and dredging apparatus," Japanese Patent JP, 1998-183678, A, July 14, 2008.

[5] Y. Tsuchiya, "Removal mechanism and method of sediments in dams and lakes," Japanese Patent JP, 2001-164543, A, June 19, 2008.

[6] N. Tajima, M. Sadatomi, A. Kawahara, and S. Terada, "Dredging of sediment in dam utilizing siphonage with sliding outer tube," Japanese Journal of Multiphase Flow, vol. 24, pp. 70-76, April 2010.

[7] T. Fukano, "Measurement of time varying thickness of liquid film flowing with high speed gas flow by a constant electric current method (CECM)," Nuclear Engineering and Design, vol. 184, pp. 363-377, August 1998.

[8] The Japan Society of Mechanical Engineers, Handbook of Gas-Liquid Two-Phase Flow Technology, Tokyo: Corona Publishing, 1989, pp. 60-72.

[9] The Japan Society of Mechanical Engineers, Hydraulic Resistance in Pipes and Ducts, 1st ed. Tokyo: The Japan Society of Mechanical Engineers, 1979, pp. 73-75.

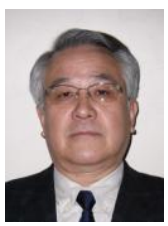

M. Sadatomi received the master degree of engineering from Kumamoto University, Japan in 1976 and became a research assistant in the same university. He received the doctor degree of engineering from Kyushu University, Japan in 1986. Now, he is a professor of the Dept. of Advanced Mechanical Systems, Graduate School of Science and Technology at Kumamoto University, and also Kyushu branch president of the Japan Society of Mechanical Engineers. His specialty is fluids engineering, especially multi-phase flow in power and environmental engineering.

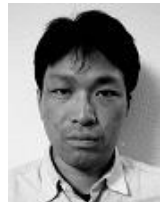

T. Nagano received the master degree of engineering from Kumamoto University, Japan in 2012 with the supervision by Prof. M. Sadatomi. Now, he is an engineer of Mitsubishi Electric Co.

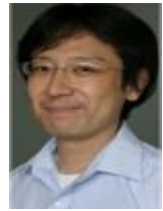

A. Kawahara received the master degree of engineering from Kumamoto University, Japan in 1990 and became a research assistant in the same university. He received the doctor degree of engineering from Kumamoto University in 1998. Now, he is an associate professor of the Dept. of Advanced Mechanical Systems, Graduate School of Science and Technology at Kumamoto University. His specialty is fluids engineering concerned with multiphase flow, especially in micro and mini channels. 